\title{
Guilda de visitantes florais de quatro espécies simpátricas de Convolvulaceae: composição e comportamento ${ }^{1}$

\author{
Guild of floral visitors \\ in four sympatric species of Convolvulaceae: \\ composition and behavior ${ }^{1}$
}

\author{
Joicelene Regina Lima da PaZ ${ }^{2}$ \\ $\&$ Camila Magalhães Pigozzo ${ }^{3}$
}

Comumente como lianas e trepadeiras, os membros da família Convolvulaceae ocorrem frequentemente em ambientes antropizados, áreas abertas, cultivos agrícolas e em bordas de fragmentos florestais. Com distribuição cosmopolita, a família Convolvulaceae exibe maior diversidade nos trópicos (JUDD et al., 2009), sendo o Brasil um importante centro de endemismo do grupo. Apesar de sua diversidade ser ainda pouco conhecida e subestimada (BurIL \& Alves, 2011), atualmente estima-se que cerca de 18 gêneros e 300 espécies sejam ocorrentes no Brasil (Souza \& LoRenzI, 2008), nas mais diversas formações vegetacionais.

As flores de Convolvulaceae apresentam características morfológicas típicas, independentes do gênero as quais pertencem, tais como: flores coloridas e vistosas, gamopétalas, frequentemente infundibiliformes ou campanuladas (SouZA \& LORENZI, 2008). As flores são efêmeras (durando um dia ou até mesmo poucas horas), geralmente exibindo antese diurna, com a abertura das flores nas primeiras horas da manhã. Comumente as flores oferecem néctar e pólen como recursos florais, e são altamente atrativas a diferentes grupos de visitantes florais, especialmente no que se refere aos insetos (JUDD et al., 2009).

${ }^{1}$ Parte da monografia da primeira autora. ${ }^{2}$ Laboratório de Entomologia, Departamento de Ciências Biológicas, Universidade Estadual de Feira de Santana (UEFS), Av. Universitária, s/n, Cidade Universitária, CEP. 44.031-460, Feira de Santana, BA, Brasil. (joicelene.lima@yahoo.com.br) (Autora correspondente). ${ }^{3}$ Centro Universitário Jorge Amado (UNIJORGE), Av. Luiz Viana Filho, Paralela, CEP. 41.745-130, Salvador, BA, Brasil. (camilapigozzo@yahoo.com.br). 
Em virtude da similaridade morfológica, atributos florais semelhantes podem levar ao compartilhamento de visitantes florais ou até mesmo a competição por polinizadores, o que pode diminuir as chances de polinização e sucesso reprodutivo da planta. Na literatura, vários estudos clássicos relatam a partilha temporal e/ou espacial de visitantes florais em espécies simpátricas, a fim de aumentar o fitness reprodutivo, assim como relatado em comunidades de Acácias africanas (STONE et al., 1996; 1998; 1999) e mexicanas (RAINE, 2001; RAINE et al., 2002), e com espécies de Convolvulaceae (Wolfe \& Sowell, 2006; Pick \& Schlindwein, 2011).

Apesar da similaridade morfológica, características como antese, disponibilidade do recurso e cor das flores podem dizer muito sobre os polinizadores potenciais. Por exemplo, em Convolvulaceae as flores polinizadas por borboletas são diurnas, róseas e de tubo estreito (MACHADO \& SAZIMA, 1987); as polinizadas por beija-flores exibem antese diurna, cor vermelha e tubo estreito (MACHADO \& SAZIMA, 1987); enquanto que as polinizadas por mariposas esfingídeos abrem à noite, têm coloração branca e tubo estreito (Willmott \& Búrquez, 1996; Galetto \& Bernardello, 2004; McMullen, 2009). Entretanto, a maioria das espécies que apresentam flores polinizadas por abelhas exibem antese diurna, coloração azulada, rósea, lilás-púrpura com o tubo floral mais alargado (Piedade, 1998; Maimoni-Rodella \& Yanagizawa , 2007; Galetto et al., 2002; Singh et al., 2010; Pick \& SCHLIndweIn, 2011).

Desta maneira, o presente estudo tem como objetivo identificar as guildas de visitantes florais e polinizadores potenciais de quatro espécies simpátricas de Convolvulaceae em um remanescente urbano de Mata Atlântica, bem como avaliar se há sobreposição na guilda de espécies visitantes.

\section{MATERIALEMÉTODOS}

ÁrEA DE ESTUDO - O estudo foi realizado no remanescente urbano de Mata Atlântica do $19^{\circ}$ Batalhão de Caçadores (BC) — Pirajá, localizado no município de Salvador, Bahia, Nordeste do Brasil (1257'53" S e $\left.38^{\circ} 27^{\prime} 14^{\prime \prime} \mathrm{W}\right)$. O fragmento abrange uma área de 240 ha, com vegetação típica de Mata Atlântica, atualmente apresentando mata secundária sob forte influência antrópica, apesar do aparente bom estado de conservação do fragmento (MACEDO et al., 2007). O clima da região é tropical quente e úmido (Af na tipologia climática de Köppen), sem estação seca bem definida. A temperatura média anual fica em torno de $25,3^{\circ} \mathrm{C}$ (MACEDO et al., 2007) e a precipitação anual de aproximadamente 2.098,7 mm (DEFESA CivIL, 2011). 
EsPÉCIES ESTUDADAS - Na área de estudo, a família Convolvulaceae é representada por quatro espécies e dois gêneros, sendo Ipomoea o mais representativo, com três espécies e Merremia com apenas uma espécie (SANTOS et al., 2009). As espécies trepadeiras ocorriam ao longo da borda da mata e das trilhas do fragmento, utilizando como suporte outras espécies, galhos e até mesmo árvores secas.

As espécies consideradas neste estudo, Ipomoea asarifolia (Desr.) Roem. \& Schult., Ipomoea bahiensis Willd. ex Roem. \& Schult., Ipomoea eriocalyx Meisn. e Merremia dissecta var. edentada (Meisn.) O’Donell, são trepadeiras herbáceas, que podem alcançar de 2 a 6 metros de altura do solo, a depender do suporte que utilizam.

ASPECTOS DA MORFOLOGIA E BIOLOGIA FLORAL E FLORAÇ̃̃o - Para cada espécie, flores frescas $(\mathrm{n}=10)$ foram fixadas em álcool $70 \%$, para posterior análise em laboratório. Os diâmetros e o comprimento das flores e das estruturas reprodutivas foram mensurados em dez flores frescas. O tamanho das flores foi classificado de acordo com MACHADO \& LOPES (2004), a forma floral seguiu LoRENZI \& GonÇALVES (2007) e a cor, o odor, o tipo de recurso e a sua localização nas flores foram determinados em campo. A presença de pigmentos ultravioleta foi verificada com flores (n $=5$ ) expostas ao vapor de hidróxido de amônio (PA), durante 30 segundos (Scogin et al., 1977). A presença de osmóforos foi realizada a partir de flores $(\mathrm{n}=3)$ imersas em vermelho neutro $(1 \%)$, durante 10 minutos (DAFNI et al., 2005).

O momento de disponibilidade do pólen, o horário de abertura, fechamento e o tempo de duração das flores foram registrados no mínimo em três flores, para cada uma das espécies em estudo. A receptividade estigmática foi testada com a imersão de estigmas $(\mathrm{n}=3)$ em peróxido de hidrogênio $\left(\mathrm{H}_{2} \mathrm{O}_{2}\right)$ (DAFNI et al., 2005), desde a fase de botão em préantese e a cada $1 \mathrm{~h}$ durante a duração das flores, ao longo dos meses de dezembro de 2007 e janeiro de 2008. A viabilidade polínica foi aferida através de anteras imersas em solução de vermelho neutro (1\%), desde a fase de botão e a cada $1 \mathrm{~h}$ durante a duração da flor $(\mathrm{n}=5)$, e posteriormente analisada em microscopia óptica (DAFNI et al., 2005). A intensidade da floração foi categorizada de acordo com os critérios de Fournier (1974) e a duração seguiu Newstrom et al. (1994).

Sistema REPRODUtivo - Para análise da biologia reprodutiva foram realizados os tratamentos de autopolinização espontânea, autopolinização manual, polinização cruzada manual e em condições naturais (controle), em no mínimo cinco flores de cada espécie. As flores foram previamente ensacadas, e quando necessárias emasculadas, sendo acompanhadas até a formação dos frutos, em cada espécie. O sucesso dos tratamentos foi calculado pela proporção de frutos/flores de cada espécie. 
VISITANTES FLORAIS - A coleta e observação dos visitantes florais foram realizadas por um coletor, mensalmente, entre dezembro de 2007 e abril de 2008. As observações iniciaram a partir das 5:30 h até o fechamento completo das flores, perfazendo um total de 533 horas de coleta e observação para cada espécie botânica. O comportamento dos visitantes foi definido através de observações visuais diretas, vídeos e fotografias. As espécies visitantes que apresentaram apenas um indivíduo foram excluídas da lista de visitantes.

A constância dos visitantes foi determinada através da fórmula: $\mathrm{C}=$ $\left(\mathrm{n}^{\circ}\right.$ de meses em que a espécie $X$ foi coletada $/ \mathrm{n}^{\circ}$ de meses totais coletados) x 100. A partir dos valores obtidos, as espécies foram categorizadas em $\mathrm{w}=$ constante $(\mathrm{C}>50 \%), \mathrm{y}=$ acessória $(\mathrm{C}=>25 \%<50 \%)$ e $\mathrm{z}=$ acidental $(\mathrm{C}<25 \%)$ (adaptado de Thomazini \& Thomazini, 2002). Para a análise da comparação múltipla da comunidade guilda visitante entre as espécies botânicas foi empregada a análise de agrupamento usando o índice de similaridade de de Sorensen (Bray-Curtis), e a técnica de aglomeração por pesos proporcionais (UPGMA), utilizando o PcOrd for Windows versão 4.25 (McCunE \& MEFFord, 1999).

Para os visitantes mais abundantes de cada espécie vegetal foram confeccionadas cinco lâminas semipermanentes, com gelatina glicerinada, para a análise da carga polínica. As lâminas confeccionadas foram depositadas na Coleção Didática do Laboratório de Botânica da Unijorge. Com base nos critérios de abundância, comportamento de coleta, contato das estruturas reprodutivas e análise polínica foram aferidos os potenciais polinizadores de cada espécie de planta.

Os visitantes florais testemunhos encontram-se depositados na Coleção Entomológica Prof. Johann Becker do Museu de Zoologia (MZFS) da Universidade Estadual de Feira de Santana (UEFS) e na coleção de referência do Laboratório de Bionomia, Biogeografia e Sistemática de Insetos (BIOSIS) da Universidade Federal da Bahia (UFBA). Os vouchers botânicos estão depositados nos Herbários Radam Brasil do Jardim Botânico de Salvador (HRB 55.467, 55.468, 55.469 e 55.470), da Universidade Estadual de Feira de Santana (HUEFS 159.591, 159.593, 159.594, 159.592) e Maria Eneyda P. Kauffmann Fidalgo (SP 420.334, $420.335,420.330,420.329)$, sob os respectivos números de tombo.

\section{RESULTADOS}

As quatro espécies de Convolvulaceae estudadas apresentam características florais semelhantes, tais como flores grandes, bissexuadas, gamopétalas, do tipo infundibiliformes (nas espécies de Ipomoea) e afunilada (em Merremia macrocalyx var. edentada). As dimensões 
florais variaram pouco entre as espécies em estudo, e os valores estão apresentados na Tabela 1. As flores apresentam coloração rósea, violeta, lilás e branca, em I. asarifolia, I. bahiensis, I. eriocalyx e M. dissecta var. edentada, respectivamente. Há presença de pigmentos ultravioleta nas corolas de todas as espécies de Ipomoea estudadas e nas anteras de M. dissecta var. edentada. A presença de osmóforos só foi observada em I. eriocalyx, no interior do tubo floral e rodeando o nectário.

A antese de todas as espécies estudadas é diurna, com as flores das espécies de Ipomoea abrindo nas primeiras horas da manhã (entre 4:00 e 5:30 h) e fechando próximo ao meio-dia, ao passo que as flores de Merremia var. edentada exibem abertura e fechamento mais tarde, às 8:00 h. As flores de todas as espécies em estudo foram consideradas efêmeras, durando aproximadamente sete horas. O pólen encontra-se disponível desde o momento da abertura das flores.

O estigma e as anteras estão receptivos e viáveis, respectivamente, durante todo o período da vida da flor, desde o botão em pré-antese até a senescência floral, em todas as espécies. Em $M$. dissecta var. edentada foram observados pequenos orifícios localizados entre os filetes, na base das flores. Em campo, observou-se que poucas horas após a abertura das flores de $M$. dissecta var. edentada, as anteras exibiam-se completamente retorcidas, no sentido helicoidal.

As características da morfologia e biologia floral de cada espécie estão apresentadas na Tabela 1.

Tabela 1. Características florais de quatro espécies de Convolvulaceae, em um remanescente urbano de Mata Atlântica, em Salvador, Bahia, Brasil. Est. = estames; Co = corola; média \pm DP.

\begin{tabular}{|c|c|c|c|c|}
\hline Características florais & Ipomoea asarifolia & Ipomoea bahiensis & Ipomoea eriocalyx & $\begin{array}{l}\text { Meremia dissecta var. } \\
\text { edentada }\end{array}$ \\
\hline $\begin{array}{l}\text { Corola (Co): } \\
\text { Forma } \\
\text { Coloração } \\
\text { Cor do tubo floral (interior) }\end{array}$ & $\begin{array}{l}\text { infundibiliforme } \\
\text { rosa } \\
\text { rosa/magenta-escuro }\end{array}$ & $\begin{array}{l}\text { infundibiliforme } \\
\text { violeta } \\
\text { púrpura }\end{array}$ & $\begin{array}{l}\text { infundibiliforme } \\
\text { lilás } \\
\text { violeta-escuro }\end{array}$ & $\begin{array}{l}\text { funil } \\
\text { branca } \\
\text { amarelo-claro }\end{array}$ \\
\hline $\begin{array}{l}\text { Dimensões: } \\
\text { Diâmetro Co }(\mathrm{mm}) \\
\text { Comprimento Co }(\mathrm{mm}) \\
\text { Abertura do tubo floral }(\mathrm{mm}) \\
\text { Estigma (comprimento) }(\mathrm{mm}) \\
\\
\text { Estames (comprimento) }(\mathrm{mm})\end{array}$ & $\begin{array}{l}60,0 \pm 1,5 \\
50,5 \pm 0,5 \\
10,0 \pm 0,6 \\
20,0 \pm 0,3 \\
\text { (2 est.) } 18,0 \pm 0,1 ; \\
\text { (3 est.) } 26,0 \pm 0,8\end{array}$ & $\begin{array}{l}50,0 \pm 0,6 \\
43,0 \pm 1,0 \\
10,0 \pm 0,4 \\
20,0 \pm 0,3 \\
\text { (2 est.) } 17,0 \pm 0,2 ; \\
(2 \text { est.) } 20,0 \pm 0,2 ; \\
(1 \text { est.) } 25,0 \pm 0,3\end{array}$ & $\begin{array}{l}50,0 \pm 0,5 \\
40,0 \pm 1,0 \\
9,3 \pm 0,4 \\
20,0 \pm 0,6 \\
\\
\text { (5 est.) } 18,00 \pm 1,3\end{array}$ & $\begin{array}{l}40,0 \pm 0,5 \\
35,0 \pm 0,5 \\
8,5 \pm 0,5 \\
25,0 \pm 0,2 \\
\text { (2 est.) } 14,0 \pm 0,3 \mathrm{e} \\
\text { (3 est.) } 22,0 \pm 0,3\end{array}$ \\
\hline $\begin{array}{l}\text { Flores/inflorescência } \\
\text { Osmóforos } \\
\text { Pigmento ultravioleta } \\
\text { Guias estruturais }\end{array}$ & $\begin{array}{l}1-6 \\
\text { Ausentes } \\
\text { Presentes Co } \\
\text { Ausentes }\end{array}$ & $\begin{array}{l}1-6 \\
\text { Ausentes } \\
\text { Presentes Co } \\
\text { Ausentes }\end{array}$ & $\begin{array}{l}2-6 \\
\text { Presente } \\
\text { Presentes Co } \\
\text { Ausentes }\end{array}$ & $\begin{array}{l}1-5 \\
\text { Ausentes } \\
\text { Presentes nas anteras } \\
\text { Presente entre os filetes }\end{array}$ \\
\hline $\begin{array}{l}\text { Antese } \\
\text { Abertura das flores } \\
\text { Fechamento das flores } \\
\text { Duração das flores }\end{array}$ & $\begin{array}{l}\text { Diurna } \\
4: 00 \mathrm{~h}-5: 30 \mathrm{~h} \\
11: 00 \mathrm{~h}-12: 00 \mathrm{~h} \\
\sim 7 \text { horas }\end{array}$ & $\begin{array}{l}\text { Diurna } \\
4: 30 \mathrm{~h}-5: 30 \mathrm{~h} \\
11: 00 \mathrm{~h}-12: 00 \mathrm{~h} \\
\sim 7 \text { horas }\end{array}$ & $\begin{array}{l}\text { Diurna } \\
5: 00 \mathrm{~h} \\
12: 00 \mathrm{~h} \\
\sim 7 \text { horas }\end{array}$ & $\begin{array}{l}\text { Diurna } \\
8: 00 \mathrm{~h} \\
14: 00 \mathrm{~h} \\
-6 \text { horas }\end{array}$ \\
\hline
\end{tabular}


As espécies das convolvuláceas estudadas floresceram simultaneamente, durante todos os meses estudados, com exceção do mês de fevereiro de 2008 , onde I. bahiensis não apresentou flores. Houve variação na intensidade do florescimento das espécies, sem aparente relação com a pluviosidade, sendo que os meses com os maiores valores de intensidade floral foram em dezembro de 2007 (I. bahiensis), janeiro de 2008 (I. eriocalyx e M. dissecta var. edentada) e dezembro-janeiro (I. asarifolia) (Fig. 1).

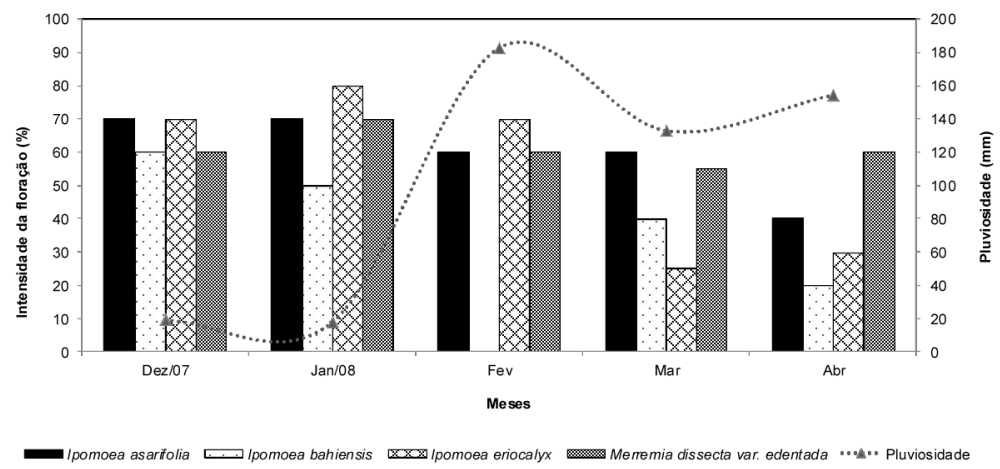

Fig. 1. Floração, em nível de população, de quatro espécies de Convolvulaceae e pluviosidade, entre dezembro de 2007 a abril de 2008, em um remanescente urbano de Mata Atlântica, Salvador (BA), Brasil.

As quatro espécies em estudo foram consideradas autocompatíveis, apresentando formação de frutos em todos os tratamentos realizados. Entretanto foram verificadas as maiores taxas de sucesso nos experimentos de polinização cruzada manual e no tratamento controle (condições naturais). Os resultados dos experimentos de biologia reprodutiva estão apresentados na Tabela 2.

As flores das plantas estudadas foram visitadas por diferentes grupos de insetos, como moscas, borboletas e besouros. Entretanto, em todas as espécies botânicas as abelhas foram os visitantes predominantes. Considerando todas as espécies de plantas em estudo, foram coletados 272 indivíduos, distribuídos em 13 espécies. Todas as espécies de visitantes florais e a sua respectiva abundância estão listadas na Tabela 3.

Em I. asarifolia foram coletados 21 visitantes florais, distribuídas entre 4 espécies de abelhas. Melitoma aff. segmentaria (OliveIRA et al., no prelo) $(\mathrm{n}=7)$ foi a espécie mais abundante e constante ao longo do período amostral, seguida das abelhas Ceratina (Crewella) spp. (OliveIRA et al., no prelo) (n =7), consideradas espécies acessórias. Enquanto a abelha Trigona spinipes $(\mathrm{n}=5)$ foi considerada um visitante acidental das flores de I. asarifolia (Tabela 3). 
Tabela 2. Resultados dos experimentos de biologia reprodutiva realizados em quatro espécies de Convolvulaceae, em um remanescente urbano de remanescente urbano de Mata Atlântica, Salvador (BA), Brasil. Flores/Frutos formados. $(\mathrm{S}=$ Sucesso).

\begin{tabular}{lccccccccc}
\hline Tratamento & I.asarifolia & I. bahiensis & $\begin{array}{c}\text { I. eriocalyx } \\
\end{array}$ & & & & & \multicolumn{2}{c}{$\begin{array}{c}\text { M.dissecta } \\
\text { var. edentata }\end{array}$} \\
\hline & N & S \% & N & S \% & N & S \% & N & S \% \\
\hline Autopolinização espontânea & $63 / 45$ & 71,4 & $39 / 28$ & 71,8 & $23 / 18$ & 78,3 & $63 / 41$ & 65,1 \\
Autopolinizaçãomanual & $40 / 29$ & 72,5 & $21 / 18$ & 85,7 & $21 / 14$ & 66,7 & $28 / 17$ & 60,7 \\
Polinização cruzadamanual & $19 / 15$ & 78,9 & $23 / 19$ & 82,6 & $05 / 04$ & 80,0 & $23 / 21$ & 91,3 \\
Condições natural/Controle & $19 / 14$ & 73,7 & $18 / 15$ & 83,3 & $19 / 17$ & 89,5 & $18 / 14$ & 77,8 \\
& & & & & & & & & \\
\hline
\end{tabular}

As flores de I. bahiensis foram visitadas por abelhas, totalizando 19 indivíduos, pertencentes a 5 espécies distintas. Dentre os visitantes florais as abelhas Ceratina (Crewella) spp. $(\mathrm{n}=9)$ foram as mais abundantes, com espécies consideradas constantes e acessórias ao longo dos meses. As demais espécies de visitantes florais foram consideradas acidentais e acessórias, mas foram pouco abundantes (Tabela 3).

Ipomoea eriocalyx apresentou 54 espécimes de visitantes florais, pertencentes a 11 espécies. Destes, as abelhas $(n=40$ e 7 espécies $)$ foram os mais representativos, seguidos de borboletas ( $n=08$ e 3 espécies) e moscas ( $n=6$ e 1 espécie) (Tabela 3). Dentre os visitantes, apenas as abelhas Ceratina (Crewella) sp. n. 1 (OliveIRA et al., no prelo) $(\mathrm{n}=11)$ e $M$. aff. segmentaria $(\mathrm{n}=11)$ destacaram-se pela abundância e constância nas flores.

Em flores de $M$. dissecta var. edentada foram coletados 178 espécimes, pertencentes a 6 espécies de abelhas. Trigona fuscipennis $(\mathrm{n}=130)$ foi a espécie mais abundante, sendo considerada acessória ao longo do estudo. As demais abelhas Trigona (T. braueri, $\mathrm{n}=08$ e $T$. spinipes, $\mathrm{n}=17$ ) foram consideradas constantes. As demais espécies visitantes exibiram pouca representatividade numérica (Tabela 3 ).

Em relação à similaridade da comunidade visitante entre as flores das plantas em estudo, apenas (Ceratina (Crewella) sp. n. 1 e Ceratina (Crewella) sp. n. 2 (OliveIRA et al., no prelo) foram comuns às quatro espécies botânicas estudadas, sendo classificadas como visitantes constantes ou acessórios ao longo dos meses, nas espécies em estudo (Tabela 3). Os demais visitantes florais foram exclusivos de apenas uma 
Tabela 3. Visitantes florais de quatro espécies de Convolvulaceae, em um remanescente urbano de Mata Atlântica, em Salvador, Bahia, Brasil, entre dezembro de 2007 e abril de 2008. $\mathrm{N}$ = número de indivíduos coletados; $\mathrm{Fr}=$ frequência relativa; $\mathrm{C}=$ constância; $\mathrm{w}=$ constante; $\mathrm{y}=$ acessória; $\mathrm{z}=$ acidental; $\mathrm{R}=$ recurso coletado; $\mathrm{Ne}=$ néctar; $\mathrm{Po}=$ pólen.

\begin{tabular}{|c|c|c|c|c|c|c|c|c|c|c|c|c|}
\hline \multirow{2}{*}{ Insetos visitantes } & \multicolumn{3}{|c|}{ Ipomoea asarifolia } & \multicolumn{3}{|c|}{ Ipomoea bahiensis } & \multicolumn{3}{|c|}{ Ipomoea eriocalyx } & \multicolumn{3}{|c|}{$\begin{array}{l}\text { Merremia dissecta } \\
\text { var. edentada }\end{array}$} \\
\hline & $\mathrm{N} / \mathrm{Fr}$ & $\mathbf{C}$ & $\mathbf{R}$ & $\mathrm{N} / \mathrm{Fr}$ & $\mathbf{C}$ & $\mathbf{R}$ & $\mathrm{N} / \mathrm{Fr}$ & $\mathbf{C}$ & $\mathbf{R}$ & $\mathrm{N} / \mathrm{Fr}$ & $\mathbf{C}$ & $\mathbf{R}$ \\
\hline $\begin{array}{l}\text { DIPTERA } \\
\text { Diptera sp. } 1\end{array}$ & -- & - & -- & $04 / 21,1 \%$ & $\mathrm{z}$ & $\mathrm{Ne}$ & $06 / 11,0 \%$ & y & $\mathrm{Ne}$ & -- & $\overline{-}$ & -- \\
\hline $\begin{array}{l}\text { LEPIDOPTERA } \\
\text { Hesperiidae }\end{array}$ & & & & & & & & & & & & \\
\hline Hesperiidae sp. 1 & -- & - & -- & --- & - & -- & $03 / 5,6 \%$ & w & $\mathrm{Ne}$ & -- & - & -- \\
\hline Hesperiidae sp. 2 & & & & --- & - & --- & $03 / 5,6 \%$ & y & $\mathrm{Ne}$ & -- & - & -- \\
\hline $\begin{array}{l}\text { Hesperiidae sp. } 3 \\
\text { HYMENOPTERA } \\
\text { Apidae } \\
\text { Meliponini }\end{array}$ & -- & - & -- & --- & - & -- & $02 / 3,7 \%$ & $\mathrm{z}$ & $\mathrm{Ne}$ & --- & - & -- \\
\hline Plebeia sp. & -- & - & -- & -- & - & -- & $05 / 9,3 \%$ & w & $\mathrm{Ne}$ & $17 / 9,6 \%$ & $z$ & $\mathrm{Ne}$ \\
\hline Trigona braueri (Friese, 1900) & -- & - & -- & -- & - & -- & -- & - & -- & $08 / 4,5 \%$ & w & Po \\
\hline Trigona fuscipennis (Friese, 1900) & -- & - & -- & -- & - & -- & $02 / 3,7 \%$ & $\mathrm{z}$ & $\mathrm{Ne}$ & $130 / 73,0 \%$ & y & Po \\
\hline Trigona spinipes (Fabricius, 1793) & $05 / 23,8 \%$ & $\mathrm{z}$ & $\mathrm{Ne}$ & --- & - & -- & $03 / 5,6 \%$ & $\mathrm{z}$ & $\mathrm{Ne}$ & $17 / 9,6 \%$ & $\mathrm{w}$ & Po \\
\hline Emphorini & & & & & & & & & & & & \\
\hline Melitoma aff. segmentaria (Fabricius, 1804) & $07 / 33,4 \%$ & w & $\mathrm{Po} / \mathrm{Ne}$ & --- & - & -- & $11 / 20,3 \%$ & w & $\mathrm{Po} / \mathrm{Ne}$ & --- & -- & -- \\
\hline $\begin{array}{l}\text { Xylocopini } \\
\text { Ceratina (Crewella) sp. } 1 \text { sp. n. } \\
\text { Ceratina (Crewella) sp. } 2 \text { sp. } \mathbf{n} .\end{array}$ & $\begin{array}{l}04 / 19,0 \% \\
05 / 23,8 \%\end{array}$ & $\begin{array}{l}\mathrm{y} \\
\mathrm{y}\end{array}$ & $\begin{array}{l}\mathrm{Po} / \mathrm{Ne} \\
\mathrm{Po} / \mathrm{Ne}\end{array}$ & $\begin{array}{l}03 / 15,8 \% \\
06 / 31,5 \%\end{array}$ & $\begin{array}{l}\mathrm{y} \\
\mathrm{w}\end{array}$ & $\begin{array}{l}\mathrm{Po} / \mathrm{Ne} \\
\mathrm{Po} / \mathrm{Ne}\end{array}$ & $\begin{array}{l}11 / 20,3 \% \\
03 / 5,6 \%\end{array}$ & $\begin{array}{l}\mathrm{w} \\
\mathrm{y}\end{array}$ & $\begin{array}{l}\mathrm{Po} / \mathrm{Ne} \\
\mathrm{Po} / \mathrm{Ne}\end{array}$ & $\begin{array}{l}04 / 2,2 \% \\
02 / 1,1 \%\end{array}$ & $\begin{array}{l}\mathrm{w} \\
\mathrm{y}\end{array}$ & $\begin{array}{l}\mathrm{Ne} \\
\mathrm{Ne}\end{array}$ \\
\hline Halictidae & & & & & & & & & & & & \\
\hline $\begin{array}{l}\text { Halictidae sp. } \\
\text { Augochlorini }\end{array}$ & -- & - & -- & $03 / 15,8 \%$ & y & $\mathrm{Po} / \mathrm{Ne}$ & $05 / 9,3 \%$ & w & $\mathrm{Po} / \mathrm{Ne}$ & --- & - & -- \\
\hline Augochlora sp. 1 sp. n. & -- & - & --- & $03 / 15,8 \%$ & $\mathrm{y}$ & $\mathrm{Po} / \mathrm{Ne}$ & -- & - & --- & --- & - & -- \\
\hline Número total de individuos & 21 & -- & --- & 19 & -- & --- & 54 & - & --- & 178 & -- & --- \\
\hline
\end{tabular}

das plantas ou de no máximo três (Tabela 3). A sobreposição da comunidade de visitantes florais nas quatro espécies de Convolvulaceae estudadas estão representadas na figura 2.

Considerando todos os meses, as visitas dos insetos ocorreram praticamente durante todo o período em que as flores permaneceram abertas, variando entre os picos de atividade diária nas quatro plantas estudadas (Fig. 3). Independentemente da espécie botânica, os visitantes florais mais abundantes foram também os mais representativos no período de maior atividade de visitação às flores. Em I. asarifolia o intervalo com o maior número de visitas foi entre 8:00 e 10:00 h (Fig. 3A). Ipomoea bahiensis teve o maior pico de visitação entre 6:00 e 8:00 h (Fig. 3B). A maioria das visitas em I. eriocalyx foram concentradas no intervalo das 8:00 às 11:00 (Fig. 3C). Enquanto, que nas flores de M. dissecta var. edentada a atividade diária foi mais representativa entre 10:00 h e 12:00 h (Fig. 3D). 


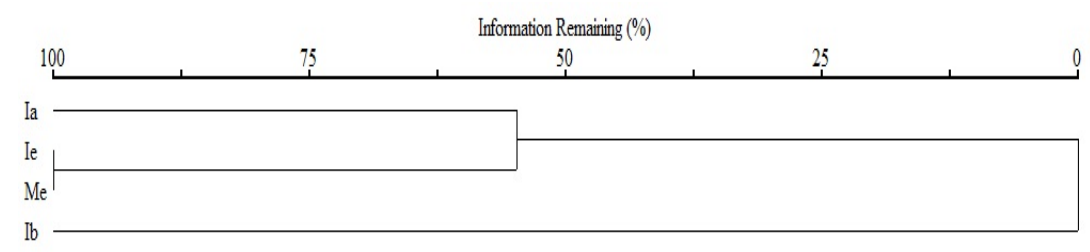

Fig, 2. Dendrograma de similaridade (\%) comparando as comunidades de visitantes florais das quatro espécies simpátricas de Convolvulaceae, entre os meses de dezembro de 2007 a abril de 2008, em um remanescente urbano de Mata Atlântica, Salvador (BA), Brasil. Ia = Ipomoea asarifolia $; \mathrm{Ie}=$ Ipomoea eriocalyx $; \mathrm{Me}=$ Merremia dissecta var. edentada $; \mathrm{Ib}=$ Ipomoea bahiensis.

O comportamento dos visitantes das flores diferiu em virtude do recurso coletado. Em coleta de néctar, as borboletas pousavam na corola e inseriam a probóscide no tubo, acessando assim o néctar. Os demais visitantes (abelhas e moscas) pousavam na corola e dirigiam-se ao final do tubo floral, inserindo o aparelho bucal diretamente no nectário. $\mathrm{Na}$ coleta de pólen as abelhas manuseavam as anteras com as pernas anteriores.

E, eventualmente, em $M$. dissecta var. edentada, as abelhas Trigona spp., utilizavam o aparelho bucal para coleta polínica. Apenas as abelhas mais abundantes e constantes de cada espécie botânica (Tab. 2) apresentavam frequentemente grãos de pólen aderidos ao corpo.

Nas abelhas que coletavam pólen das flores de Ipomoea spp. ( $M$. aff. segmentaria, Ceratina (Crewella) spp. e Halictidae sp.) o pólen ficava aderido na região ventral do tórax do visitante. Ao passo que, nas abelhas visitantes de $M$. dissecta var. edentada a presença de pólen restringia-se á região da cabeça e tórax (dorsal), podendo assim contatar as estruturas reprodutivas florais.

Comumente, as abelhas do gênero Trigona além de coletarem pólen e néctar, também coletavam partes florais de $M$. dissecta var. edentada, especialmente das pétalas de flores abertas e botões em pré-antese. As mordeduras na corola das flores não raramente chegavam a destruir parciais ou até mesmo totalmente a corola.

Para cada espécie de planta em estudo, a análise carga polínica dos principais visitantes florais apresentou baixa riqueza de tipos polínicos diferentes. E, adicionalmente, observou-se também, boa representatividade do morfotipo polínico específico, em cada uma das plantas estudadas (Tabela 4). 

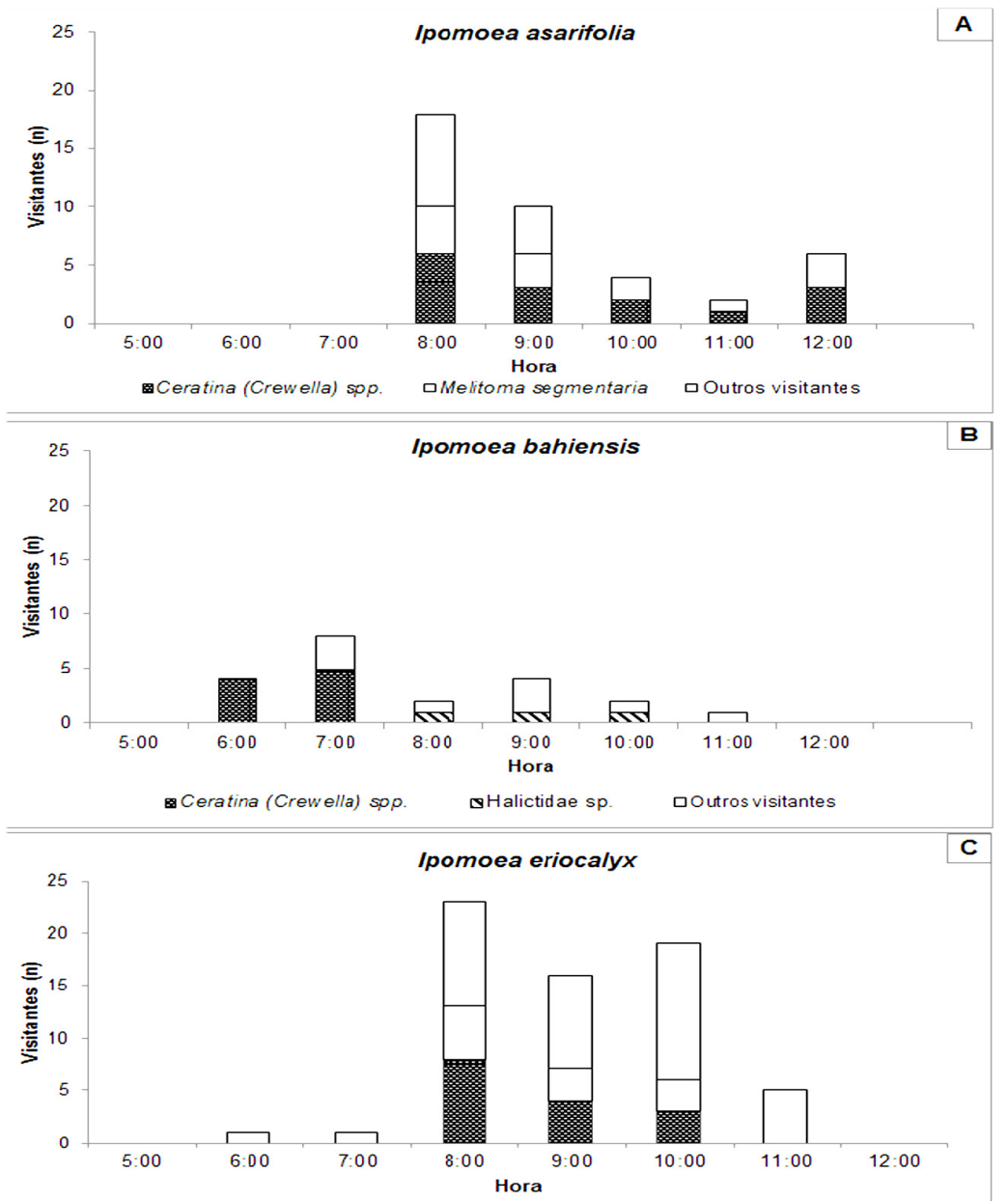

Q Ceratina (Crewella) spo. 口Melitoma segmentaria 口Outros visitantes

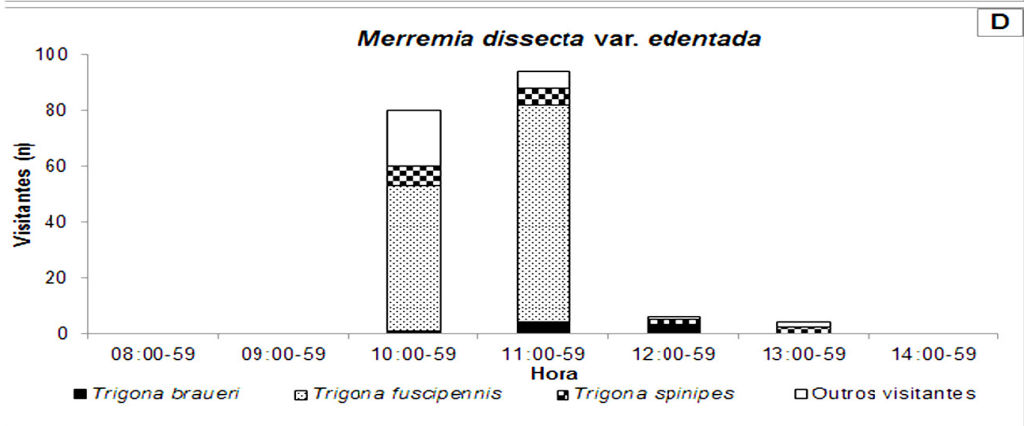

Fig. 3. Atividade diária dos visitantes florais em flores de Convolvulaceae, entre os meses de dezembro de 2007 a abril de 2008, em um remanescente urbano de Mata Atlântica, Salvador (BA), Brasil. 
Tabela 4. Análise da carga polínica dos polinizadores potenciais de espécies de Convolvulaceae, em um remanescente urbano de remanescente urbano de Mata Atlântica, Salvador, Bahia, Brasil.

\begin{tabular}{lllll}
\hline Espécie & Visitante & Local & TP & RP \\
\hline Ipomoea asarifolia & Melitoma aff. segmentaria $(\mathrm{n}=5)$ & Tórax (ventral) & 02 & $93,7 \%$ \\
& Ceratina $($ Crewella $)$ spp. $(\mathrm{n}=5)$ & Tórax (ventral) & 02 & $78,3 \%$ \\
Ipomoea bahiensis & Ceratina $($ Crewella $)$ spp. $(\mathrm{n}=2)$ & Tórax (ventral) & $02 \quad 85,0 \%$ \\
& Halictidae sp. $(\mathrm{n}=3)$ & Tórax (ventral) & $03 \quad 85,0 \%$ \\
Ipomoea eriocalyx & Ceratina $($ Crewella $)$ spp. $(\mathrm{n}=5)$ & Tórax (ventral) & 04 & $72,3 \%$ \\
& Melitoma aff. segmentaria $(\mathrm{n}=5)$ & Tórax (ventral) & $01100,0 \%$ \\
Merremia dissecta & Trigona spp. (n $=5$ & Tórax (cab. e dorso) $02 \quad 75,0 \%$ \\
var. edentada & & & &
\end{tabular}

[ABREVIATURAS $-\mathrm{TP}=$ Tipo polínico, $\mathrm{RP}=$ Representatividade polínica.

\section{DISCUSSÃO}

As flores das espécies de Convolvulaceae em estudo apresentam características que as enquadram na síndrome da melitofilia (cf. FAEGRI \& VAN DER PIJL, 1979). Na literatura, a melitofilia é uma síndrome bastante relatada para convolvuláceas, inclusive associada às espécies dos gêneros Ipomoea (Piedade, 1998; Galetto \& Bernadello, 2004; Maimoni-Rodella \&Yanagizawa, 2007) e Merremia (Neves et al., 2006; RAIMúnDEZ-URRUTIA et al., 2008; PICK \& SCHLINDWEIN, 2011). Apesar de haver registros, ainda que poucos, de outras síndromes de polinização, tais como a psicofilia (Machado \& Sazima, 1987), ornitofilia (Machado \& Sazima, 1987; Wolfe \& Sowell, 2006), e esfingofilia (Willmott \& Búrquez, 1996; McMullen, 2009; PAZ, 2011).

A coloração floral é um importante atrativo visual aos visitantes florais. Em Ipomoea spp., as corolas das flores variaram entre os tons de rosa, violeta e lilás. Cores bem relatadas para o gênero, que comumente apresenta flores conspícuas e vistosas (BIANCHINI, 2009). Ao passo que, 
flores alvas e de tons mais claros são bem típicos dos representantes de Merremia (BIANChINI, 2009). A presença de pigmentos ultravioleta na corola das flores de Ipomoea e nas anteras de M. dissecta var. edentada também podem atuar como um guia visual, funcionando como um atrativo aos visitantes florais, especialmente às abelhas, que conseguem enxergar essa faixa do espectro de luz (RoubiK, 1989).

As flores de Ipomoea são do tipo infundibiliformes, com o tubo floral alongado e maior abertura no ápice, enquanto que as flores de $M$. dissecta var. edentada apresentam-se em forma de funil, com tubo e abertura mais curtos. Assim, as diferenças morfológicas das flores no que refere à forma, comprimento e diâmetro do tubo floral podem ter influenciado a guilda de animais visitantes, bem como a forma de coleta dos recursos. As flores, como a maioria dos membros de Convolvulaceae oferecem pólen e néctar como recursos florais (SouZA \& LoRENZI, 2008).

Sendo efêmeras, as flores exibem antese diurna, corroborando com outros estudos onde as flores abrem nas primeiras horas da manhã em Ipomoea (PIEDADE, 1998) e mais tarde, no meio da manhã em Merremia (Austin, 1982). Adicionalmente, a presença de flores de todas as espécies durante todos os meses observados, com exceção de I. bahiensis em fevereiro de 2008, podem oferecer maior atratividade aos animais. Além disso, a coexistência das espécies simpátricas floridas simultaneamente, podem propiciar maior atratividade aos animais, bem como, funcionar como mancha de recurso, favorecendo a manutenção da comunidade de visitantes locais (STONE et al., 1996; 1998; 1999).

Apesar da autocompatibilidade observada nas espécies de Convolvulaceae, as maiores taxas de formação de frutos foram registradas nos tratamentos de polinização cruzada e em condições naturais (controle). Segundo SERES \& RAMíREZ (1995) a sincronia no horário da antese favorece o aumento do número de flores disponíveis, e aumenta as chances de polinização cruzada em plantas. Nas flores analisadas, a disposição e proximidade das estruturas reprodutivas, ocorrendo em mesmo nível, podem favorecer a autopolinização nas espécies observadas, assim como sugerido por KIILL \& RANGA (2003) em estudos com I. asarifolia. Além disso, a receptividade e viabilidade das estruturas reprodutivas positivas durante toda a vida da flor, nas quatro espécies analisadas também podem favorecer a autofecundação das espécies.

A autocompatibilidade das espécies analisadas às tornam independentes do vetor de pólen para promover a polinização. MAIMONIRodella \& Rodella (1982) também menciona autocompatibilidade em M. dissecta var. edentada em ambiente antropizado, no Sudeste do Brasil. 
Entretanto, diferentemente dos resultados obtidos, outros autores relatam sistemas de autoincompatibilidade em I. asarifolia (KILL \& RANGA, 2003) e I. bahiensis (PIEDADE, 1998; PACHECo Filho et al., 2011), com todos os estudos realizados no sertão de Pernambuco.

Assim, desta maneira, uma das possíveis explicações para o distinto comportamento destas espécies seria a plasticidade reprodutiva, que pode ser influenciada por fatores abióticos, como o ambiente em que as espécies estão inseridas, e até mesmo por fatores bióticos, como a ausência da disponibilidade de polinizadores (MEDRANo et al., 1999), por exemplo.

As abelhas foram predominantemente, os principais visitantes das flores de Convolvulaceae na área de estudo. A morfologia floral pouco restritiva e a facilidade ao acesso dos recursos florais também possibilitaram visitas de outros grupos de insetos às flores, tais como moscas, borboletas.

A atração de uma comunidade diversificada de visitantes parece ser um comportamento comum entre convolvuláceas. Outros estudos também relatam a presença de vários animais nas flores (ex. borboletas, moscas, mariposas, beija-flores, besouros dentre outros). Mas, os principais visitantes são as abelhas (PIEDAdE, 1998; MAIMONI-Rodella \& YANAGIZAWA, 2007; Raimúndez-UrRutia et al., 2008; Pick \& Schlindwein, 2011). O comportamento de coleta observado também é muito semelhante ao descrito em outras convolvuláceas estudadas (PIEDADE, 1998; PICK \& SchlindweIn, 2011), especialmente no que refere às abelhas.

O pico de visitação das flores foi diferenciado entre as plantas analisadas. Geralmente ocorrendo mais cedo nas espécies de Ipomoea e mais tarde em Merremia. Entretanto, apesar das diferenças no pico, em todas as plantas, as espécies mais importantes de abelhas estavam ativas nas respectivas flores. Apesar de não ter sido observado, outros estudos realizados com espécies simpátricas mencionam a separação temporal e espacial de visitantes através de diferenças no horário da antese ou disponibilidade de recursos, amadurecimento das estruturas reprodutivas, deiscência das anteras ou ainda diferenças no pico de visitação das flores (STONE et al., 1996; 1998; 1999).

O presente estudo exibiu maior riqueza e diversidade de espécies visitantes quando comparados com outros estudos mais duradouros com as mesmas espécies em outros ambientes (MaImoni-Rodella \& Rodella, 1982; Piedade, 1998; Pacheco Filho et al., 2011). A guilda visitante verificada nos estudos de KIILL \& RANGA (2003) em I. asarifolia, não exibiu espécies comuns com presente estudo. Nos estudos de Piedade (1996) e PACHECo FiLHo et al. (2011) foram comuns abelha Augochlora sp. e borboletas Hesperiidae spp. em flores de I. bahiensis. Em flores de $M$. dissecta var. edentada, também foram observadas visitas de 
besouro, T. spinipes e Augochlora spp. (Maimoni-Rodella \& Rodella, 1982).

Apenas duas espécies de abelhas (Ceratina (Crewella) sp. 3 sp. n. e Ceratina (Crewella) sp. 4 sp. n.) (OLIVEIRA et al., no prelo) foram comuns às quatro espécies botânicas, sendo importantes visitantes em relação à frequiência às flores durante os meses em estudo. Esses resultados sugerem que apesar das semelhanças nas características florais em espécies que coexistem na mesma região, isso não resultou em uma guilda de visitantes similar. Em outros estudos onde autores avaliaram a partição e o compartilhamento de visitantes florais em espécies simpátricas de Convolvulaceae, também foi observado pouca sobreposição da comunidade de visitantes, com poucas espécies de animais comuns às espécies botânicas analisadas (ver Wolfe \& Sowell, 2006; PICK \& SCHLINDWEIN, 2011).

Além disso, para o comportamento da baixa sobreposição da comunidade de visitantes florais entre as espécies deve ser avaliada pelo ponto de vista da área de estudo ser um fragmento urbano. E, assim sendo, a ausência de dados de diversidade e abundância de visitantes disponíveis não nos permite comparar se a comunidade local também é alterada.

Em virtude do comportamento, constância e abundância as abelhas foram consideradas os potenciais polinizadores das quatro espécies analisadas. Além de exibirem a alta fidelidade floral, as abelhas contatavam as estruturas reprodutivas. Adicionalmente, imagina-se que a ocorrência de estames com comprimentos diferentes e o comportamento de coleta, especialmente das abelhas que adentram no tubo floral e manipulam as anteras, podem favorecer o contato entre as estruturas, promovendo assim, a polinização (PIEDADE, 1998).

No que se refere às espécies de abelhas da tribo Emphorini, incluindo M. segmentaria, possuem hábito oligolético, e geralmente está associado às flores de convolvuláceas, o que pode justificar a alta fidelidade floral da espécie (Zanella, 2000; Pinheiro \& Schlindwein, 2011). Assim, estudos de visitantes florais em nível de comunidade são importantes, para o conhecimento da fauna local e da guilda de visitantes de espécies aparentadas filogeneticamente podem servir de subsídio do conhecimento do sistema reprodutivo das espécies e de análises filogenéticas e biogeográficas das interações dos polinizadores.

Em I. asarifolia e I. eriocalyx as abelhas Melitoma aff. segmentaria e do subgênero Crewella spp. foram considerados os polinizadores potenciais. Nas flores de I. bahiensis foram as abelhas do subgênero Crewella spp. e Halictidae sp. Ao passo que, em M. dissecta var. 
edentada foram às abelhas Trigona spp. os polinizadores potenciais. Alguns autores sugerem que possa existir uma relação entre abelhas oligoléticas da tribo Emphorini (como Melitoma segmentaria) e flores de Convolvulaceae, especialmente em representantes de Ipomoea (Zanella, 2000; Pick \& Schlindwein, 2011).

Em $M$. dissecta var. edentada, abelhas Trigona ssp. foram observadas constantemente destruindo parcial ou totalmente a corola das flores. Segundo VIEIRA et al. (2007) esta abelha apresenta mandíbulas fortes e bastante desenvolvidas, por isso vários autores consideram abelhas deste gênero, especialmente Trigona spinipes (Fabricius, 1793) (Almeida \& Laroca, 1988; Boiça Junior et al., 2004; Azeredo et al., 2006; Pereira, 2008). Como pragas, estas abelhas por exibirem o forrageamento e a exploração de recursos muito agressiva, com o hábito de destruir partes de plantas cultivadas, como flores, folhas, hastes e frutos, assim como observado neste estudo.

No presente estudo, as características da morfologia e biologia florais foram semelhantes entre as convolvuláceas analisadas. De maneira geral, as plantas estiveram floridas durante todos os meses, propiciando maior atratividade floral e promovendo a manutenção de polinizadores locais. As flores oferecem néctar e pólen como recursos, de acesso facilitado. Em virtude disso, as flores foram atrativas a vários grupos de insetos, especialmente às abelhas, polinizadores potenciais das quatro espécies em estudo.

Além disso, a capacidade de autofecundação e a ocorrência de mais de uma espécie como polinizador potencial pode ser uma característica vantajosa, especialmente para espécies de plantas ruderais, que ocorrem com freqüência em ambientes alterados ou na colonização de novos ambientes cuja fauna visitante também é alterada ou pode ser incerta.

\section{RESUMO}

As flores de Convolvulaceae apresentam características morfológicas peculiares. Acredita-se que atributos florais semelhantes podem levar ao compartilhamento ou competição de visitantes florais. Tornando-se imprescindível o desenvolvimento de estratégias vegetais a fim de aumentar o sucesso reprodutivo, minimizando a partilha de visitantes e polinizadores. O presente estudo objetiva identificar as guildas de visitantes florais e polinizadores potenciais quatro espécies simpátricas de Convolvulaceae em um remanescente urbano de Mata Atlântica, bem como avaliar se há sobreposição na guilda de espécies visitantes. Para tal, visitantes florais e a fenologia reprodutiva foram acompanhadas mensalmente, entre dezembro de 2007 a abril de 2008. De modo geral, 
as características da morfologia e biologia florais foram semelhantes entre as convolvuláceas analisadas. As plantas estiveram floridas praticamente durante todos os meses, propiciando maior atratividade floral, promovendo a manutenção de polinizadores locais. As flores apresentam antese diurna e oferecem néctar e pólen como recursos, de acesso facilitado. Em virtude disso, as flores foram atrativas a vários grupos de insetos, especialmente às abelhas que são os polinizadores potenciais das quatro espécies. As plantas são autocompatíveis, e a ocorrência de mais de uma espécie como polinizador potencial pode ser uma característica vantajosa, especialmente para espécies de plantas simpátricas que ocorrem com freqüência em ambientes alterados cuja fauna visitante também é alterada ou pode ser incerta.

PALAVRAS-CHAVE: abelhas; Ipomoea; Merremia; plantas-pioneiras; trepadeiras

\section{ABSTRACT}

The flowers of Convolvulaceae show typical morphological characteristics. Similar floral traits can lead to sharing or competition of

floral visitors and potential pollinators. Therefore becoming essential to develop strategies to enhance plant reproductive success by minimizing the number of visitors and pollinators shared. Thus, this study aims to identify the guilds of floral visitors and potential pollinators four sympatric species of Convolvulaceae in an urban remnant of Atlantic Forest, and to assess whether there is overlap in the guild of species visitors. To this end, reproductive phenology and floral visitors were monitored monthly from December 2007 to April 2008. In general morphological characteristics and biological the flowers were similar to the species Convolvulaceae analyzed. The plants have been flowering during practically every month, providing greater floral attractiveness, promoting the maintenance of local pollinators. The flowers have diurnal anthesis and offer nectar and pollen as resources, easy access. As a result, the flowers are attractive to various groups of insects, especially bees that are potential pollinators of four species. The plants are selfcompatible, and the occurrence of more than one species potential pollinator may be an advantageous characteristic, especially for plant species sympatric frequently occur in altered environments which the animals visits also changed, or may be uncertain.

KEYwORDS: bees, Ipomoea, Merremia, pioneer plants, vines. 


\section{RÉSUME}

Les fleurs de Convolvulaceae présentent caractéristiques morphologiques typiques, indépendante du genre à laquelle elles appartiennent. Ainsi, on croit que les traits floraux similaires peuvent conduire à des visiteurs partage ou la compétition florale. Ce qui rend essentiel pour élaborer des stratégies visant à améliorer le succès de reproduction de la plante, ce qui minimise le partage de visiteurs et les pollinisateurs. Ainsi, cette étude vise à identifier les guildes de visiteurs floraux et les pollinisateurs potentiels de quatre espèces sympatriques de Convolvulaceae dans un vestige de urbaine de Forêt Atlantique, ainsi d'évaluer s'il existe un chevauchement entre la guilde des espèces visitant. À cette fin, les visiteurs floraux et phénologie de la reproduction ont été suivis tous les mois de Décembre 2007 to Avril 2008. En général, les caractéristiques de la biologie florale et de la morphologie étaient similaires entre les Convolvulaceae analysées. Les plants qui fleurissaient pendant presque tous les mois, en fournissant plus attrayant floral, en favorisant le maintien des pollinisateurs locaux. Les fleurs présentent anthèse diurne et offre le nectar et le pollen des fonctionnalités pour faciliter l'accès. En conséquence, les fleurs étaient attrayants pour les différents groupes d'insectes, notamment les abeilles, qui sont les pollinisateurs potentiels de ces quatre espèces. Les plantes sont auto-compatibles, et la présence de plus d'une espèce comme pollinisateur potentiel peut être une caractéristique avantageuse surtout pour les espèces végétales simpatric qui se produisent changeant fréquemment environnements dans lesquels le visiteur est également modifié la faune ou peut être incertain.

Mots-CLÉs: abeilles; Ipomoea: Merremia; pionnières; plantes-grimpantes.

\footnotetext{
Agradecimentos - As autoras agradecem aos membros do 19 $\mathrm{BC}$ - Batalhão Pirajá, pela autorização do acesso à área de estudo. Ao MSc. Erivaldo Pereira Queiroz (JBSSA-BA) pela identificação inicial e à Prof. Dr ${ }^{a}$. Rosângela Simão Bianchini (IBT-SP) pela confirmação da espécie botânica. À Prof ${ }^{a}$. Dr ${ }^{a}$. Favízia F. de Oliveira (UFBA-BA) e ao MSc. Thiago Mahlmann V. L. Muniz (INPA-AM) pela identificação das abelhas. À mestranda Thamara Zacca Bispo Taumaturgo (UFPR-PR) pela identificação e morfotipificação dos lepidópteros. À Unijorge-BA, representando pelo Prof ${ }^{\circ}$. Edinaldo Luz das Neves, pelos empréstimos de equipamentos e materiais de campo e laboratório. Á Mayanne Jesus Oliveira (UFBA-BA) pela ajuda e colaboração em todas as etapas deste estudo, e aos demais colegas pela colaboração e ajuda no campo e em laboratório. Aos revisores anônimos pelas relevantes sugestões e críticas ao manuscrito.
} 


\section{BIBLIOGRAFIA}

Almeida, M. C. \& S. Laroca. 1988. Trigona spinipes (Apidae, Meliponinae): taxonomia, bionomia e relações tróficas em áreas restritas. Acta Biológica Paranaense 17: 67-108.

Austin, D. F. 1982. Convolvulaceae. Pp. 15-239. In.: Z. L. Febres \& J. A. Steyermark (eds.) Flora da Venezuela. Fundación Educación Ambiental. Caracas, 8 (3).

Azeredo, E. H.; E. Lima \& P. C. R. Cassino. 2006. Ocorrência de Trigona spinipes (Fabr., 1793) (Hymenoptera: Apidae, Meliponinae) em resposta à fatores climáticos e doses de nitrogênio e potássio em duas cultivares de batateira. Revista da Universidade Rural Série Ciências da Vida 26: 10-23.

BianchinI, R. 2009. Flora de Grão-Mogol, Minas Gerais: Convolvulaceae. Bolm Bot Univers São Paulo 27: 33-41.

Boiça Júnior, A. L.; T. M. Santos \& J. Passilongo. 2004. Trigona spinipes (Fabr.) (Hymenoptera: Apidae) em espécies de maracujazeiro: flutuação populacional, horário de visitação e danos às flores. Neotr. Entomol. 33: 135-139.

Buril, M. T. \& M. Alves. 2011. Flora da Usina São José, Igarassu, Pernambuco: Convolvulaceae. Rodriguésia 62: 093-105.

Dafni, A.; P. G. Kevan \& B. C. Husband (eds.). 2005. Practical pollination biology. Enviroquest, Ltd, Cambridge, Ontario, Canada. 590pp.

Defesa Civil De Salvador. 2011. Disponível em <http:// w w w. d e f e s a c i vil.s a l v a d o r. b a.g o v. b r / index.php?option=com_content $\&$ task=view $\&$ id=43\&Itemid $=54$. (Acesso em 08/09/11)>.

Faegri, K. \& L. VAn Der PiJl. 1979. The principles of pollination ecology. Third Edition. Pergamon Press. Oxford. 244 pp.

Fournier, L. A. 1974. Un metodo cuantitativo para la medición de caracteristicas fenológicas en arboles. Turrialba 24: 422-423.

Galetto, L. \& G. Bernardello. 2004. Floral nectaries, nectar production dynamics and chemical composition in six Ipomoea species (Convolvulaceae) in relation to pollinators. Annals of Botany 94: 269280.

Galetto, L.; A. Fioni \& A. Calviño. 2002. Éxito reproductivo y calidad de los frutos en poblaciones del extremo sur de la distribución de Ipomoea purpurea (Convolvulaceae). Darwiniana 40: 25-32.

Judd, W. S.; C. S. Campbell; E. A. Kellogg; P. F. Stevens \& M. J. Donoghue. 2009. Sistemática vegetal: um enfoque filogenético. $3^{\mathrm{a}}$ Edition. Artmed, Porto Alegre. 612 pp. 
KiILl, L. H. P. \& N. T. RanGa. 2003. Ecologia da polinização de Ipomoea asarifolia (Ders.) Roem. and Schult. (Convolvulaceae) na região semi-árida de Pernambuco. Acta Botanica Brasilica 17: 355-362.

LORENZI, H. \& A. G. GonÇALVEs. 2007. Morfologia vegetal: organografia e dicionário ilustrado de morfologia das plantas vasculares. Nova Odessa: Instituto Plantarum de estudos da flora. São Paulo. 446 pp.

Macedo, T. S.; L. L. Fernandes; D. F. Silva; A. S. Varjão; A. S. Neves \& C. M. Pigozzo. 2007. Comparação florística entre um fragmento de Mata Atlântica e ambientes associados (Restinga e Manguezal) na cidade de Salvador, Bahia. Revista Virtual Candombá 3: 138148.

MACHADO, I. C. S. \& A. V. Lopes. 2004. Floral traits and pollination systems in the Caatinga, a Brazilian tropical dry forest. Annals of Botany 94: 365-376.

Machado, I. C. S. \& M. SAzima. 1987. Estudo comparativo da biologia floral em duas espécies invasoras: Ipomoea hederifolia e I. quamoclit (Convolvulaceae). Revta brasil. Bot. 47: 425-436.

Maimoni-Rodella, R. C. S. \& Y. Yanagizawa. 2007. Floral biology and breeding system of three Ipomoea weeds. Planta Daninha 25: 3542.

Maimoni-Rodella, R. C. S. \& R. A. Rodella. 1982. Aspectos da biologia floral de Merremia dissecta (Jacq.) Hall. f. var. edentata (Meissn.) O'Donell (Convolvulaceae). Revta Agric. 61: 213-222.

Mccune, B. \& M. J. MefFord. 1999. Multivariate Analysis of Ecological Data. Version 4.25.

MCMULLEN, C. K. 2009. Pollination biology of a night-ûowering galápagos endemic Ipomoea habeliana (Convolvulaceae). Bot. Journ. Linnean Soc. 160: 11-20.

Medrano, M.; Guttián, P.; J. Guitián. 1999. Breeding system and temporal variation in fecundity of Pancratium maritimum L. (Amarylliaceae). Flora, 194: 13-19.

Neves, E.L.; H. Taki; F. O. Silva; B. F. Viana \& P. G. Kevan. 2006. Flower characteristics and visitors of Merremia macrocalyx (Convolvulaceae) in the Chapada Diamantina, Bahia, Brazil. Lundiana 7:97-102.

Newstrom, L. E.; G. W. Frankie \& H. G. Baker. 1994 A new classification for plant phenology based on flowering patterns in lowland tropical rain forest trees at La Selva, Costa Rica. Biotropica 26: 141-159.

Pacheco Filho, A. J. S.; C. Westerkamp \& B. M. Freitas. 2011. Ipomoea bahiensis pollinators: Bees or butterûies? Flora 206: 662—667. 
PAz, J. R. L. DA. 2011. Biologia Floral e Polinização Diurna e Noturna de Ipomoea carnea subsp. fistulosa (Mart. ex Choisy) D.F. Austin (Convolvulaceae) em uma Área Antropizada no Semi-árido da Bahia, Brasil. Dissertação de Mestrado. Universidade Estadual de Feira de Santana. Feira de Santana, BA, Brasil. 35 pp.

Pereira, D. S. 2008. Estudo do potencial de produção de néctar da jitirana-branca (Merremia aegyptia) em área de Caatinga no sertão central em Quixeramobim-CE. Dissertação de mestrado. Universidade Federal Rural do Semi-Árido. Mossoró, RN, Brasil. 75 pp.

Pick, R.A. \& C. Schlindwein. 2011. Pollen partitioning of three species of Convolvulaceae among oligolectic bees in the Caatinga of Brazil. Plant Syst. Evolution 293:147-159.

Piedade, L.H. 1998. Biologia da polinização e reprodutiva de sete espécies de Convolvulaceae na caatinga no sertão de Pernambuco. Tese de doutorado. Universidade Estadual de Campinas. Campinas, SP, Brasil. 123 pp.

Raimúndez-Urrutia, E.; Avendaño, L. \& D. Velázquez. 2008. Reproductive biology of the morning glory Merremia macrocalyx (Ruiz \& Pavon) O'Donnell (Convolvulaceae). Journ. Torrey Botanical Soc. 135: 299-308.

RAINE, N. E. 2001. The pollination ecology of a mexican Acacia da comunidad. PhD Thesis. University of Oxford. Oxford, UK.

Raine, N. E.; P. G. Willmer \& G. N. Stone. 2002. Spatial structuring and floral avoidance behaviour prevent ant-pollinator conflict in a Mexican ant-acacia. Ecology 83: 3086-3096.

RoubiK, D. W. 1989. Ecology and natural history of tropical bees. 1. ed. Cambridge, Cambridge University Press, $514 \mathrm{pp}$.

Santos, M. F. O.; E. P. Queiroz \& C. M. Pigozzo. 2009. Síndromes de polinização em fragmento urbano de Mata Atlântica do $19^{\circ}$ Batalhão de Caçadores, Cabula, Salvador, Bahia. Revista Virtual Candombá 5: 26-39.

Scogin, R.; D. A. Young \& C. E. Jones. 1977. Anthochlor pigments and pollination biology: II. The ultraviolet patterns of Coreopsis gigantea (Asteraceae). Bulletin Torrey Botanical Club 104: 155-159.

SERES, A. \& N. RAMIREz. 1995. Biologia floral y polinización de algunas monocotiledoneas de un bosque nublado Venezolano. Annals Missouri Botan. Garden 82:61-81.

Singh, K. P.; G. Bhavana \& G. Dhakre. 2010. Biologia Reprodutiva de Evolvulus L. alsinoides (Medicinal Herb). International Journal of Botany 6: 304-309. 
SouzA, V. C. \& H. LoRenzi. 2008. Botânica Sistemática: guia ilustrado para identificação das famílias brasileiras de fanerógamas nativas e exóticas no Brasil, baseado em APG II. Second Edition. Nova Odessa: Instituto Plantarum de estudos da flora São Paulo. 703 pp.

Stone, G. N.; P. G. Willmer \& S. Nee. 1996. Daily partitioning of pollinators in an African Acacia community. Proc. Royal Society B 263: 13891393.

Stone, G. N.; P. G. Willmer \& J. A. Rowe. 1998. Partitioning of pollinators during flowering in an African Acacia community. Ecology 79: 28082827.

Stone, G. N.; P. G. Willme; J. A. Row; B. Nyundo \& R. Abdallah. 1999. The pollination ecology of Mkomazi Acacia species. Pp. 337-360. In.: M.J. Coe, N. Mcwilliam, G. N. Stone, M. Packer (eds.). Mkomazi, the ecology, biodiversity and conservation of a Tanzanian savanna. The Royal Geographical Society: London.

Thomazini, M. J. \& A. P. B. W. Thomazini. 2002. Diversidade de abelhas (Hymenoptera: Apoidea) em inflorescências de Piper hispidinervum (C. DC.). Neotr. Entomol. 31: 27-34.

Vieira, C. U.; C. M. Rodovalho; L. O. Almeida; A. C. S. Siquieroli \& A. M. BonetTi. 2007. Interação entre Trigona spinipes Fabricius, 1793 (Hymenoptera: Apidae) e Aethalion reticulatum Linnaeus, 1767 (Hemiptera: Aethalionidae) em Mangifera indica (Anacardiaceae). Journ. Biosci. 23: 10-13.

Willmott, A. P. \& A. Búrquez. 1996. The pollination of Merremia palmeri (Convolvulaceae): can hawk moths be trusted? Am. Jour. Botany 83: 1050-1056.

Wolfe, L. M. \& D. R. Sowell. 2006 Do pollination syndromes partition the pollinator community? A test using four sympatric morning glory species. Intern. Journ. Plant Sciences 167: 1169- 1175.

Zanella, F. C. V. 2000. The bees of the Caatinga (Hymenoptera, Apoidea, Apiformes): A species list and comparative notes regarding their distribution. Apidologie 31: 579-592.

Recebido em 25 de agosto de 2012. 